OITS-578

\title{
MULTIPLICITY DISTRIBUTIONS OF SQUEEZED ISOSPIN STATES
}

\author{
I. M. Dremin* and Rudolph C. Hwa ${ }^{\dagger}$ \\ Institute of Theoretical Science and Department of Physics, \\ University of Oregon, Eugene, Oregon 97403-5203
}

\begin{abstract}
Multiplicity distributions of neutral and charged particles arising from squeezed coherent states are investigated. Projections onto global isospin states are considered. We show how a small amount of squeezing can significantly change the multiplicity distributions. The formalism is proposed to describe the phenomenological properties of neutral and charged particles anomalously produced in hadronic and nuclear collisions at very high energies.
\end{abstract}

Typeset using REVTEX

*Permanent address: P. N. Lebedev Physical Institute, Leninsky prospect, 53, Moscow 117924 Russia. Electronic address: dremin@lpi.ac.ru

$\dagger$ Electronic address: hwa@oregon.uoregon.edu 


\section{INTRODUCTION}

There has been a long-standing interest in the study of the multiplicity distributions of coherent states modified by various dynamical constraints [1-6]. The recent interest in the disoriented chiral condensate (DCC) [7] as a possible explanation of the Centauro-type events [8] has further extended the use of coherent states in the study of unusual charge distributions [9]. It has also been suggested [10,11] that a natural quantum description of the DCC can be given in terms of the squeezed states [12 [15]. In this paper we investigate the neutral and charge distributions of squeezed coherent states constrained to form global isospin scalar and vector states that can possibly arise in nuclear collisions at very high energies. Our emphasis will be on the multiplicity distributions of the charged and neutral pions. It is therefore a significantly more detailed investigation than would be required, if the goal is only to determine the average fraction of neutral particles produced.

If charged pions are always produced in pairs to maintain local charge neutrality of the source medium, it is very natural to consider squeezed states, which are created from the vacuum by operators that are exponentiations of the quadratic forms of the creation and annihilation operators. Although the squeezed states are constructed in quantum optics to reduce noise in certain applications $[12$ [15], their mathematical structure has features that are useful in quantizing classical fields. Their relevance to hadron production in high-energy collisions was recognized long ago [4]. Recent interest in the subject has been stimulated by the study of chiral phase transition, where the long wavelength pion mode is found in the linear $\sigma$ model to grow rapidly after quenching [16,17. It need not be a DCC, but the interest in developing a quantum description of DCC has led to the realization of the relevance of the squeezed states [11].

In our view that is rooted in the phenomenology of hadronic and nuclear collisions, the likelihood of the existence of a long wavelength pion mode or the creation of a DCC shielded from the normal vacuum by a hot shell in a heavy-ion collision is very remote, no matter how ideal or contrived the collision process may be. While we can think of various arguments 
against the conditions favorable for their occurrence, there has not been any serious effort made to present a phenomenologically reasonable scenario in the form of realistic modeling that can demonstrate the feasibility of their appearance in actual collisions. Thus the subject is driven so far mainly by the attractiveness of the theoretical idea and the alluring possibility of solving concrete classical equations. Until such time as when experiments suggest the relevance of disoriented chiral condensates to the anomalous production of charge and neutral particles, we shall regard the subject as an interesting speculation that does not preempt other possible approaches.

We begin with the observation of two facts. One is the empirical fact that anomalous events like the Centauro have been recorded in cosmic-ray experiments [8], though not in the laboratory. The other is the lack of a theoretical framework to describe the unusual charge distributions independent of the chiral dynamics. It seems to us useful to have a language to convey quantitatively the characteristics of the distributions without a rigid dynamical basis so that one may have what amounts to a representation in much the same way that the negative binomial distributions have been used to describe multiplicity distributions in general [4.18, [19]. While there exist earlier attempts to describe exotic neutral and charge distributions in terms of the coherent states [2, [2], it is better for our purpose to introduce some parameters that can be adjusted to change the neutral-to-charge ratio. To that end we find that the squeezed coherent states are well endowed with features suitable for our needs. Moreover, the consideration of global isotopic spin turns out to be crucial, as in [1], since unlike quantum optics the squeezed coherent states produced in hadronic and nuclear collisions must have a limited range of possible initial isotopic spin states. We should emphasize, however, that the dynamical origin of the squeezed coherent states in such collisions is not an issue that must be addressed first; we use the squeezed coherent state here only as a mathematical framework to discuss the production of neutral and charge quanta that are locally neutral in a certain limit (squeezed vacuum).

We give in Sec. II a brief review of the squeezed coherent state. Application to particle production is considered in Sec. III, and the results on multiplicity distributions are 
presented in Sec. IV.

\section{SQUEEZED STATES}

In this section we summarize the basic properties of squeezed states [12 15,20] that are to be used in the following section.

We start with the coherent state $|\alpha\rangle$, created from the vacuum by the displacement operator $D(\alpha)$ :

$$
|\alpha\rangle=D(\alpha)|0\rangle
$$

where

$$
D(\alpha)=e^{\alpha a^{\dagger}-\alpha^{*} a}
$$

$a^{\dagger}$ and $a$ are creation and annihilation operators, and $\alpha$ is a complex number. From (2.2) we have

$$
D^{\dagger}(\alpha) a D(\alpha)=a+\alpha
$$

whereupon we obtain the fundamental property that $|\alpha\rangle$, defined in (2.1), is an eigenstate of $a$

$$
a|\alpha\rangle=\alpha|\alpha\rangle
$$

with eigenvalue $\alpha$.

The squeeze operator $S(\eta)$ is defined by

$$
S(\eta)=\exp \left[\left(\eta^{*} a^{2}-\eta a^{\dagger 2}\right) / 2\right]
$$

where $\eta=r e^{i 2 \psi}$. A unitary transformation on $a$ by $S(\eta)$ yields

$$
S^{\dagger}(\eta) a S(\eta)=\mu a-\nu a^{\dagger}
$$

where 


$$
\mu=\cosh r, \quad \nu=e^{i 2 \psi} \sinh r
$$

Inverting (2.6) and a similar one on $a^{\dagger}$ gives

$$
a(\eta) \equiv S(\eta) a S^{\dagger}(\eta)=\mu a+\nu a^{\dagger}
$$

The unitary transformation of $S(\eta)$ on the displacement operator yields

$$
S^{\dagger}(\eta) D(\alpha) S(\eta)=D(\alpha(\eta))
$$

where

$$
\alpha(\eta)=\mu \alpha+\nu \alpha^{*}
$$

The squeeze state $|\alpha, \eta\rangle$ is defined by

$$
|\alpha, \eta\rangle=D(\alpha) S(\eta)|0\rangle
$$

Evidently, when $\eta=0$, i.e. no squeezing, $|\alpha, 0\rangle$ is identical to the coherent state $|\alpha\rangle$. Even for $\eta \neq 0$, the expectation value of $a$ is still $\alpha$, since we have, using (2.3) and (2.6)

$$
\langle\alpha, \eta|a| \alpha, \eta\rangle=\left\langle 0\left|S^{\dagger}(\eta)(a+\alpha) S(\eta)\right| 0\right\rangle=\alpha
$$

However, $|\alpha, \eta\rangle$ is not an eigenstate of $a$ for $\eta \neq 0$; instead, it is an eigenstate of $a(\eta)$, defined in (2.8), i.e.,

$$
\begin{aligned}
a(\eta)|\alpha, \eta\rangle & =S(\eta) a S^{\dagger}(\eta) D(\alpha) S(\eta)|0\rangle \\
& =S(\eta) a|\alpha(\eta)\rangle=\alpha(\eta)|\alpha, \eta\rangle .
\end{aligned}
$$

Inverting (2.8) we have

$$
a=\mu a(\eta)-\nu a^{\dagger}(\eta)
$$

so that

$$
a^{\dagger} a=\left(\mu^{2}+|\nu|^{2}\right) a^{\dagger}(\eta) a(\eta)-\mu \nu a^{\dagger}(\eta)^{2}-\mu \nu^{*} a(\eta)^{2}+|\nu|^{2}
$$


where $\left[a(\eta), a^{\dagger}(\eta)\right]=1$ has been used. It then follows from (2.13) and (2.10) that

$$
\left\langle\alpha, \eta\left|a^{\dagger} a\right| \alpha, \eta\right\rangle=|\alpha|^{2}+|\nu|^{2}
$$

Thus the expectation value of the number operator for the squeezed state differs from that for the pure coherent state by the additive term $|\nu|^{2}$.

\section{MULTIPLICITY DISTRIBUTIONS FOR SQUEEZED ISOSPIN STATES}

We now generalize the squeezed states described in the previous section to the isospin space in a manner similar to how the coherent states are generalized in Ref. 1. The annihilation operator $\vec{a}$ is now a vector in the isospin space, and the corresponding eigenstate $|\vec{\alpha}\rangle$ is created from the vacuum by the operator $D(\vec{\alpha})$. The squeezed state is then

$$
|\vec{\alpha}, \eta\rangle=D(\vec{\alpha}) S(\eta)|0\rangle
$$

The complex vector $\vec{\alpha}=\alpha \hat{n}$ has a direction in the isospin space, specified by the polar angles $\theta$ and $\phi$. A squeezed state with a specific total isospin $I$ and $z$-component $I_{z}$ can then be expressed in terms of $|\vec{\alpha}, \eta\rangle$ in the usual way

$$
|\alpha, \eta\rangle_{I, I_{z}}=\int d \Omega_{\hat{n}} Y_{I, I_{z}}^{*}(\theta, \phi)|\vec{\alpha}(\theta, \phi), \eta\rangle
$$

What we need to do is to determine the projection of this state to the eigenstates of the number operators specifying the neutral $\left(n_{0}\right)$ and charged $\left(n_{c}\right)$ particle multiplicities.

Then the multiplicity distribution of a squeezed isospin state is

$$
P_{n_{0}, n_{c}}^{I, I_{z}}(\alpha, \eta)=\left|\left\langle n_{0}, n_{c} \mid \alpha, \eta\right\rangle_{I, I_{z}}\right|^{2}
$$

It will be parameterized by the variables $\alpha$ and $\eta$. More precisely, the results will depend only on the magnitudes $|\alpha|$ and $r$, as one might surmise from (2.16).

To proceed, it is necessary to determine first $\left\langle n_{0}, n_{c} \mid \vec{\alpha}, \eta\right\rangle$, which in turn can follow from a knowledge of the scalar product between a squeezed state and a coherent state. Following 
Yuen [12], we first note that the differential operator representation of any polynomial $M\left(\vec{a}, \vec{a}^{\dagger}\right)$ of $\vec{a}$ and $\vec{a}^{\dagger}$ in the coherent-state representation is

$$
\left\langle\vec{\beta}\left|M\left(\vec{a}, \vec{a}^{\dagger}\right)\right| \psi\right\rangle=M\left(\frac{\vec{\beta}}{2}+\frac{\partial}{\partial \vec{\beta}^{*}}, \vec{\beta}^{*}\right)\langle\vec{\beta} \mid \psi\rangle
$$

Applying this to $\vec{a}(\eta)$ for $M\left(\vec{a}, \vec{a}^{\dagger}\right)$, we have with the help of (2.13)

$$
\langle\vec{\beta}|\vec{a}(\eta)| \vec{\alpha}, \eta\rangle=\vec{\alpha}(\eta)\langle\vec{\beta} \mid \vec{\alpha}, \eta\rangle=\left[\mu\left(\frac{\vec{\beta}}{2}+\frac{\partial}{\partial \vec{\beta}^{*}}\right)+\nu \vec{\beta}^{*}\right]\langle\vec{\beta} \mid \vec{\alpha}, \eta\rangle .
$$

This is a differential equation on $\langle\vec{\beta} \mid \vec{\alpha}, \eta\rangle$ that has the solution

$$
\langle\vec{\beta} \mid \vec{\alpha}, \eta\rangle=\exp \left[-\frac{1}{2}|\vec{\beta}|^{2}-\frac{\nu}{2 \mu} \vec{\beta}^{*} \cdot \vec{\beta}^{*}+\frac{1}{\mu} \vec{\beta}^{*} \cdot \vec{\alpha}(\eta)+f\left(\vec{\alpha}(\eta), \vec{\alpha}^{*}(\eta)\right)\right] .
$$

The last term that depends only on $\vec{\alpha}(\eta)$ and $\vec{\alpha}^{*}(\eta)$ can be determined by working with

$$
\begin{aligned}
\langle\vec{\alpha}, \eta|\vec{a}(\eta)| \vec{\beta}\rangle & =\left[\frac{\vec{\alpha}(\eta)}{2} \frac{\partial}{\partial \vec{\alpha}^{*}(\eta)}\right]\langle\vec{\alpha}, \eta \mid \vec{\beta}\rangle \\
& =\left(\mu \vec{\beta}+\frac{\nu}{2} \vec{\beta}^{*}+\nu \frac{\partial}{\partial \vec{\beta}}\right)\langle\vec{\alpha}, \eta \mid \vec{\beta}\rangle .
\end{aligned}
$$

The required form for $f\left(\vec{\alpha}(\eta), \vec{\alpha}^{*}(\eta)\right)$ is therefore

$$
f\left(\vec{\alpha}(\eta), \vec{\alpha}^{*}(\eta)\right)=-\frac{1}{2}|\vec{\alpha}(\eta)|^{2}+\frac{\nu^{*}}{2 \mu} \vec{\alpha}^{2}(\eta) .
$$

Since $\vec{\beta}$ represents both the neutral and charged sectors, $\beta \cos \theta$ and $\beta \sin \theta$, in the isospin space, respectively, we have the usual representation of a coherent state $|\vec{\beta}\rangle$ in the $\left|n_{0}, n_{c}\right\rangle$ basis, i.e.,

$$
\left\langle n_{0}, n_{c} \mid \vec{\beta}\right\rangle=\frac{\beta^{n_{0}+n_{c}}}{\sqrt{n_{0} ! n_{c} !}}(\cos \theta)^{n_{0}}(\sin \theta)^{n_{c}} e^{-|\vec{\beta}|^{2} / 2} \Phi(\phi) \quad,
$$

where $\Phi(\phi)=\exp \left[i\left(n_{+}-n_{-}\right) \phi\right]$ is a phase factor that depends on the difference between + and - charged-particle multiplicities.

Using (3.6), (3.8) and (3.9) on

$$
\langle\vec{\beta} \mid \vec{\alpha}, \eta\rangle=\sum_{n_{0}, n_{c}}\left\langle\vec{\beta} \mid n_{0}, n_{c}\right\rangle\left\langle n_{0}, n_{c} \mid \vec{\alpha}, \eta\right\rangle
$$


and the expansion

$$
e^{2 \vec{z} \cdot \vec{t}-\vec{t}^{2}}=\sum_{n_{0}, n_{c}} \frac{t_{0}^{n_{0}} t_{c}^{n_{c}}}{n_{0} ! n_{c} !} H_{n_{0}}\left(z_{0}\right) H_{n_{c}}\left(z_{c}\right)
$$

where $t_{0}=t \cos \theta, t_{c}=t \sin \theta$ and similarly for $z_{0}$ and $z_{c}, H_{n}(z)$ being the Hermite polynomial, we have

$$
\left\langle n_{0}, n_{c} \mid \vec{\alpha}, \eta\right\rangle=A_{n_{0}, n_{c}}[\alpha(\eta)] H_{n_{0}}\left[\frac{\alpha_{0}(\eta)}{\sqrt{2 \mu \nu}}\right] H_{n_{c}}\left[\frac{\alpha_{c}(\eta)}{\sqrt{2 \mu \nu}}\right] \Phi(\phi)
$$

where

$$
A_{n_{0}, n_{c}}[\alpha(\eta)]=\left(n_{0} ! n_{c} !\right)^{-\frac{1}{2}}\left(\frac{\nu}{2 \mu}\right)^{\left(n_{0}+n_{c}\right) / 2} \exp \left[-\frac{1}{2}|\vec{\alpha}(\eta)|^{2}+\frac{\nu^{*}}{2 \mu} \vec{\alpha}^{2}(\eta)\right] .
$$

Note that $A_{n_{0}, n_{c}}[\alpha(\eta)]$ is independent of the isospin direction, whereas the arguments of $H_{n}$ are, since $\alpha_{0}(\eta)=\alpha(\eta) \cos \theta$ and $\alpha_{c}(\eta)=\alpha(\eta) \sin \theta$. For the isospin projected state (3.2), we obtain correspondingly

$$
\left\langle n_{0}, n_{c} \mid \alpha, \eta\right\rangle_{I, I_{z}}=A_{n_{0}, n_{c}}(\alpha, \mu, \nu) B_{n_{0}, n_{c}}^{I, I_{z}}(b)
$$

where

$$
\begin{gathered}
B_{n_{0}, n_{c}}^{I, I_{z}}(b)=\int_{-1}^{1} d \cos \theta \int_{0}^{2 \pi} d \phi Y_{I, I_{z}}^{*}(\theta, \phi) H_{n_{0}}(b \cos \theta) H_{n_{c}}(b \sin \theta) \Phi(\phi) \\
b=\alpha(\eta) / \sqrt{2 \mu \nu}
\end{gathered}
$$

When used in (3.3), this gives us the multiplicity distributions that we seek.

We have not given any special attention to the normalization of $\langle\vec{\beta} \mid \vec{\alpha}(\eta)\rangle$ as a solution to the differential equation in (3.5). That freedom will be used in normalizing $P_{n_{0}, n_{c}}^{I, I_{z}}(\alpha, \eta)$ at the end.

There is more freedom in the squeezed states than is needed in our problem. From (2.7) and (2.10) we recall that

$$
\vec{\alpha}(\eta)=\left(\alpha \cosh r+\alpha^{*} \sinh r e^{i 2 \psi}\right) \hat{n}
$$

Let us set $\alpha, \mu$, and $\nu$ all real so that 


$$
\vec{\alpha}(\eta)=\alpha(\mu+\nu) \hat{n}
$$

and

$$
b=\frac{\alpha(\mu+\nu)}{\sqrt{2 \mu \nu}} \quad, \quad \mu=\sqrt{1+\nu^{2}} .
$$

From (3.3) and (3.14) we have

$$
P_{n_{0}, n_{c}}^{I, I_{z}}(\alpha, \nu)=\frac{N}{n_{0} ! n_{c} !}\left(\frac{\nu}{2 \sqrt{1+\nu^{2}}}\right)^{n_{0}+n_{c}}\left|B_{n_{0}, n_{c}}^{I, I_{z}}(b)\right|^{2},
$$

where $N$ is a normalization factor.

The average multiplicity $\langle n\rangle$ is

$$
\langle n\rangle=\left\langle n_{0}\right\rangle+\left\langle n_{c}\right\rangle=\alpha^{2}+\nu^{2},
$$

as required by (2.16), which is invariant under rotation in the isospin space. We shall find it more convenient in the following to use the squeeze parameter $s$, defined by

$$
s=(\nu / \alpha)^{2}
$$

so that

$$
\langle n\rangle=\alpha^{2}(1+s)
$$

In all instances that we shall consider below, the value of $s$ will be small, so $\langle n\rangle$ will not be effected very much by squeezing. Nevertheless, we shall see that for certain isospin states the $n_{0}$ and $n_{c}$ distributions can be significantly influenced by the small amount of squeezing.

In the next section we shall calculate $P_{n_{0}, n_{c}}^{I, I_{z}}$ only for $I=0$ and 1 , although there exist no obstacles in carrying out the computation at higher values of $I$. For $p \bar{p}$ annihilation $I \leq 1$ is all that is necessary. For $p p$ inelastic collisions the isospin of the pion state can in principle be as large as $I=2$, but we shall restrict our consideration here to only $I \leq 1$. It is tacitly assumed that the analysis considered here is only appropriate for the particles produced in the central region. Even for nuclear collisions we hope that $I \leq 1$ is sufficient.

For $I=0,1$, we can write 


$$
Y_{I, I_{z}}(\theta, \phi)=c_{I, I_{z}}(\cos \theta)^{I-\left|I_{z}\right|}(\sin \theta)^{\left|I_{z}\right|} e^{i I_{z} \phi}
$$

Thus the $\phi$ integration in (3.15) requires $n_{+}-n_{-}=I_{z}$. For the $\theta$ integration let us define

$$
J_{n_{0}, n_{c}}^{k, l}(b)=\int_{-1}^{1} d x x^{k}\left(1-x^{2}\right)^{l / 2} H_{n_{0}}(b x) H_{n_{c}}\left(b \sqrt{1-x^{2}}\right)
$$

so that $B_{n_{0}, n_{c}}^{I, I_{z}}(b)$ is proportional to it. Let $G\left(t_{1}, t_{2}\right)$ be the generating function, from which $J_{n_{0}, n_{c}}^{k, l}$ can be obtained:

$$
J_{n_{0}, n_{c}}^{k, l}(b)=\left.\frac{\partial^{n_{0}}}{\partial t_{1}^{n_{0}}} \frac{\partial^{n_{c}}}{\partial t_{2}^{n_{c}}} G\left(t_{1}, t_{2}\right)\right|_{t_{1}=t_{2}=0}
$$

Eq. (3.25) implies

$$
\begin{aligned}
G\left(t_{1}, t_{2}\right) & =\sum_{n_{0}, n_{c}} \int_{-1}^{1} d x x^{k}\left(1-x^{2}\right)^{l / 2} H_{n_{0}}(b x) H_{n_{c}}\left(b \sqrt{1-x^{2}}\right) \frac{t_{1}^{n_{0}} t_{2}^{n_{c}}}{n_{0} ! n_{c} !} \\
& =e^{-t_{1}^{2}-t_{2}^{2}} \int_{-1}^{1} d x x^{k}\left(1-x^{2}\right)^{l / 2} e^{2 b\left(x t_{1}+\sqrt{1-x^{2}} t_{2}\right)} \\
& =e^{-t_{1}^{2}-t_{2}^{2}} \sum_{n, m=0}^{\infty}(2 b)^{n+m} \frac{t_{1}^{n} t_{2}^{m}}{n ! m !} B\left(\frac{n+k+1}{2}, \frac{m+l+2}{2}\right) \epsilon_{n+k},
\end{aligned}
$$

where $B(u, v)$ is the Euler beta function. The factor $\epsilon_{n+k}$ is 1 if $n+k=$ even, 0 if $n+k=$ odd; it is due to the symmetry of the integrand under $x \leftrightarrow-x$. It can be shown after some algebraic manipulations that

$$
\left.\frac{d^{q}}{d t^{q}}\left(e^{-t^{2}} t^{n}\right)\right|_{t=0}=(-1)^{q-n} \frac{q !}{(q-n) !} H_{q-n}(0)
$$

Since the order of $p$ of $H_{p}(z)$ must be even when $z=0, q-n$ must be even, so $(-1)^{q-n}=1$. Using this in (3.26) and (3.27) yields

$$
\begin{aligned}
J_{n_{0}, n_{c}}^{k, l}(b)= & \sum_{n=0}^{n_{0}} \sum_{m=0}^{n_{c}}(2 b)^{n+m} H_{n_{0}-n}(0) H_{n_{c}-m}(0)\left(\begin{array}{c}
n_{0} \\
n
\end{array}\right)\left(\begin{array}{c}
n_{c} \\
m
\end{array}\right) \\
& B\left(\frac{n+k+1}{2}, \frac{m+l+2}{2}\right) \epsilon_{n+k}
\end{aligned}
$$

We finally have from (3.20)

$$
P_{n_{0}, n_{c}}^{I, I_{z}}(\alpha, s)=\frac{N^{\prime}}{n_{0} ! n_{c} !}\left[4\left(1+\frac{1}{s \alpha^{2}}\right)\right]^{-\left(n_{0}+n_{c}\right) / 2}\left|J_{n_{0}, n_{c}}^{k, l}(b)\right|^{2}
$$


where $N^{\prime}$ is another normalization constant. From the exponents in (3.24) and (3.25), we have

$$
\begin{array}{lll}
I=0: & k=0, l=0, & n_{+}=n_{-} . \\
I=1, I_{z}=0: & k=1, l=0, & n_{+}=n_{-} . \\
I=1, I_{z}=1: & k=0, l=1, & n_{+}=n_{-}+1 .
\end{array}
$$

Clearly, $n_{c}=n_{+}+n_{-}$is even (odd), when $n_{+}=n_{-}\left(n_{-}+1\right)$. When $k=0, n$ must be even,

\begin{tabular}{|c|c|c|c|}
\hline (a) & $I=0:$ & $n_{0}=$ even & $n_{c}=$ even \\
\hline (b) & $I=1, I_{z}=0:$ & $n_{0}=$ odd & $n_{c}=$ even \\
\hline (c) & $I=1, I_{z}=1:$ & $n_{0}=$ even & $n_{c}=$ odd \\
\hline
\end{tabular}
so $n_{0}$ must also be even; for $k=1, n_{0}$ is odd. Thus the evenness or oddness of $n_{0}$ and $n_{c}$ can be summarized as follows:

\section{RESULTS ON MULTIPLICITY DISTRIBUTIONS}

In this section we present the results of our calculation of $P_{n_{0}, n_{c}}^{I, I_{z}}(\alpha, s)$. Our objective is to provide some insight into the dependences on squeezing and on the isospin states. To that end we shall fix the average multiplicity $\langle n\rangle$ at some reasonable value, which we take to be 25. Thus $\alpha$ and $s$ are constrained through (3.23). To demonstrate the sensitivity on squeezing for certain isospin states, we shall consider only two small values of $s$, viz., $s=0.02$ and 0.04 , which is sufficient to reveal the qualitative features of the $s$ dependence. Other higher values of $s$ can readily be considered, but are not examined here to avoid visual confusion in the results to be presented.

In calculating $P_{n_{0}, n_{c}}^{I, I_{z}}(\alpha, s)$ we note that $s$, as defined in (3.22), is independent of the sign of $\nu$. In (3.19) we have taken $\mu$ and $\nu$ to be real, but it does not preclude $\nu$ to be negative, corresponding to $\psi=\pi / 2$ in (2.7). Since the result will depend on the sign of $\nu$, we shall use the notation $s_{ \pm}$, to stand for $\operatorname{sgn}(\nu)= \pm$, while specifying the value of $s$. In the following the distributions of the neutral and charged sectors are given separately, where for fixed $\langle n\rangle$ 


$$
\begin{aligned}
& P_{n_{0}}^{I, I_{z}}\left(s_{ \pm}\right)=\sum_{n_{c}} P_{n_{0}, n_{c}}^{I, I_{z}}\left(\alpha, s_{ \pm}\right) \\
& P_{n_{c}}^{I, I_{z}}\left(s_{ \pm}\right)=\sum_{n_{0}} P_{n_{0}, n_{c}}^{I, I_{z}}\left(\alpha, s_{ \pm}\right)
\end{aligned}
$$

For $I=0$, the $n_{0}$ distribution $P_{n_{0}}^{0}\left(s_{ \pm}\right)$is insensitive to $s_{ \pm}$, but the $P_{n_{c}}^{0}\left(s_{ \pm}\right)$is highly sensitive to $s_{ \pm}$. This is shown in Figs. 1 and 2 for the cases $\nu>0$ and $\nu<0$, respectively. Clearly, $P_{n_{0}}^{0}$ is peaked at low $n_{0}$, while the peak of $P_{n_{c}}^{0}$ shifts to low $n_{c}$ with increasing $s_{-}$, although stationary in $s_{+}$. The behavior of $P_{n_{0}}^{0}$ for squeezed states is very similar to the coherent case considered in [3]. There, the asymptotic behavior is $f^{-1 / 2}$, where $f=n_{0} /\left(n_{0}+n_{c}\right)$, (obtained by using the Stirling formula in analytical estimates). Thus that law is not greatly influenced by slight squeezing.

For $I=1, I_{z}=0, \nu>0, P_{n_{0}}^{1,0}\left(s_{+}\right)$is very nearly the same as $P_{n_{c}}^{1,0}\left(s_{+}\right)$, even though the former is only for odd $n_{0}$ and the latter for even $n_{c}$, as shown in Fig. 3. Their shapes depend sensitively on $s_{+}$. For $I=1, I_{z}=0, \nu<0$, the distributions are very different; $P_{n_{0}}^{1,0}\left(s_{-}\right)$ is not sensitive to $s_{-}$, but $P_{n_{c}}^{1,0}\left(s_{-}\right)$is. That is shown in Fig. 4. Upon comparing these to $P_{n_{0}, n_{c}}^{0}$, it is evident that the distributions depend crucially on the isospin, even though $I_{z}=0$ in both cases.

Finally, for $I=1, I_{z}=1, P_{n_{0}}^{1,1}\left(s_{ \pm}\right)$behaves very similarly as $P_{n_{0}}^{0}\left(s_{ \pm}\right)$, with peaking at low $n_{0}$ and independence on $s_{ \pm}$, as is clear in Figs. 5 and $6 . P_{n_{c}}^{1,1}\left(s_{ \pm}\right)$is also similar to $P_{n_{c}}^{0}\left(s_{ \pm}\right)$, but the former is only for odd $n_{c}$, while the latter is for even $n_{c}$. Thus the $f^{-1 / 2}$ behavior is restored again.

It is relevant to ask whether the $n_{t}=n_{0}+n_{c} \rightarrow \infty$ limit should be independent of squeezing. There are two cases to consider: $s \rightarrow 0$ and $s$ finite. The $s$ dependence can be seen by examining (3.29). For infinitesimal $s, b$ is large, so the term with $n=n_{0}$ and $m=n_{c}$ is dominant. Then the large $n_{t}$ behavior is independent of the Hermite polynomials. In the $n_{t} \rightarrow \infty$ limit the $s$ dependence of (3.29) cancels that in (3.20), resulting in no net dependence on $s$. But if $s$ is set at a finite value initially, all terms in the sum in (3.29) can be important, since $H_{p}(0)$ diverges with increasing $p$. The large $n_{t}$ limit of $J_{n_{0}, n_{c}}^{k, l}$ can then 
be very different and $P_{n_{0}, n_{c}}^{I, I_{z}}$ can depend in $s$, even if $s$ is not large. In both cases, if $n_{t}$ is not infinite, there would in general be some dependence on $s$, the details of which can only be seen from the numerical results. It is for this reason that our results depend on squeezing.

To summarize, we observe that in all isospin states the $n_{c}$ distributions are most sensitive to squeezing, not just in the value of $s$, but especially in the sign of $\nu$. That is to be contrasted from the peaking of the neutral distribution $P_{n_{0}}$ at $n_{0}=0$ for both the $I=0$ state and the $I=1, I_{z}=1$ state, revealing the preference for small numbers of neutral pions for either sign of $\nu$. On the other hand, for $I=1, I_{z}=0$ the neutral to charge ratio is about 1 when $\nu>0$, but is greater than 1 when $\nu<0$. The latter therefore leans toward the characteristics of the anti-Centauro events. By varying $I, I_{z}$, and $s_{ \pm}$, one can obtain a wide variety of possible shapes of $P_{n_{c}}$ that could be used to fit the observed charged particle distribution in high-energy collision for any given average total multiplicity. In that sense its usefulness is rather similar to that of the negative binomial distribution, which has a $k$ parameter that adjusts the width. It should be noted that neither the negative binomial nor the pure squeezed-state distributions can reproduce properly the behavior of the cumulants observed in the experiments [21,22], whereas the question about squeezed isospin states is so far open. Unlike the negative binomial distribution, we now have a great deal more freedom to accommodate very anomalous production processes, where the neutral to charge ratio need not be 1:2. Indeed, $P_{n_{0}}$ can be peaked at $n_{0}=0$, which is a feature that may render this description relevant to the Centauro events. By varying the squeeze parameter, one can not only adjust the neutral to charge ratio, but also change the detailed $n_{0}$ and $n_{c}$ dependences.

We have not made any proposal in this paper to suggest that the dynamical origin for the anomalous production of particles is related to the squeezed states. We have only presented a formalism for the phenomenological description of unusual $P_{n_{0}}$ and $P_{n_{c}}$. If in the future such a formalism is found empirically to be well suited for the data, then perhaps one may be motivated to look deeper into the question of whether squeezed states are produced in certain collisions at high energy. 


\section{Acknowledgment}

We are grateful to H. Carmichael and M. Raymer for very helpful discussions on squeezed states. This work was supported in part by a NATO grant under reference CRG 930025, by the Russian Fund for Fundamental Research under grant No. 93-02-3815, and by the U.S. Department of Energy under grant No. DE-FG06-91ER40637. 


\section{REFERENCES}

[1] D. J. Scalapino and R. L. Sugar, Phys. Rev. D8, 2284 (1973); J. C. Botke, D. J. Scalapino and R. L. Sugar, Phys. Rev. D9, 813 (1974).

[2] D. Horn and R. Silver, Ann. of Physics. 66, 509 (1971).

[3] I. V. Andreev, JETP Lett. 33, 367 (1981).

[4] P. Carruthers and C. C. Shih, In. J. Mod. Phys. 2, 1447 (1987).

[5] J.-P. Blaizot and A. Krzywicki, Phys. Rev. D46, 246 (1992).

[6] R. C. Hwa and M. T. Nazirov, Phys. Rev. Lett. 68, 1988 (1992); R. C. Hwa and J. Pan, Phys. Lett. B297, 35 (1992); R. C. Hwa, Phys. Rev. C50, 383 (1994).

[7] J. D. Bjorken, In. J. Mod. Phys. A7, 4189 (1992); Acta Physica Polonica B23, 561 (1992).

[8] C. M. G. Lattes, Y. Fujimoto, and S. Hasegawa, Phys. Rep. 65, 151 (1980).

[9] K. L. Kowalski and C. C. Taylor, Case Western Reserve University Report No. 92-6, hep-ph/9211282 (unpublished); J. D. Bjorken, K. L. Kowalski and C. C. Taylor, SLACPUB-6109 (1993).

[10] I. I. Kogan, Phys. Rev. D48, R3971 (1993).

[11] R. D. Amado and I. I. Kogan, Phys. Rev. D51, 190 (1995).

[12] H. P. Yuen, Phys. Rev. A13, 2226 (1976).

[13] C. M. Caves, Phys. Rev. D23, 1693 (1981).

[14] B. L. Schumaker, Phys. Rep. 135, 317 (1986).

[15] D. F. Walls, Nature 306, 141 (1983).

[16] K. Rajagopal and G. Wilczek, Nucl. Phys. B399, 395 (1993); B404, 577 (1993). 
[17] For a review see K. Rajagopal, in Quark-Gluon Plasma 2, edited by R. C. Hwa (World Scientific, Singapore) - to be published.

[18] A. Giovannini and L. Van Hove, Z. Phys. C30, 391 (1986); Acta Phys. Polon. B19, 495 (1988).

[19] R. C. Hwa, Phys. Rev. D51, 3323 (1995).

[20] F. Haake and M. Wilken, in Photons and quantum fluctuations, edited by E. R. Pike and H. Walther (Adam Hilger, Bristol, 1988).

[21] I. M. Dremin, Physics-Uspekhi 37, 715 (1994).

[22] V. V. Dodonov, I. M. Dremin, P. G. Polynkin, and V. I. Man'ko, Phys. Lett. A193, 209 (1994). 


\section{Figure Captions}

Fig. 1 Multiplicity distributions of neutral and charged particles for $I=0$ and $\nu>0$. Open symbols are for neutral particles and full symbols are for charged particles. Circles (open and full) are for $s=0.02$; triangles (open and full) are for $s=0.04$.

Fig. 2 Same as in Fig. 1 but for $I=0, \nu<0$.

Fig. 3 Same as in Fig. 1 but for $I=1, I_{z}=0, \nu>0$. (a) and (b) are for both $n_{0}$ and $n_{c}$, but (c) is for $n_{c}$ only to show the dependence on $s$.

Fig. 4 Same as in Fig. 3 but for $I=1, I_{z}=0, \nu<0$.

Fig. 5 Same as in Fig. 1 but for $I=1, I_{z}=1, \nu>0$.

Fig. 6 Same as in Fig. 1 but for $I=1, I_{z}=1, \nu<0$. 
This figure "fig1-1.png" is available in "png" format from: http://arxiv.org/ps/hep-ph/9510223v1 
This figure "fig2-1.png" is available in "png" format from: http://arxiv.org/ps/hep-ph/9510223v1 
This figure "fig1-2.png" is available in "png" format from: http://arxiv.org/ps/hep-ph/9510223v1 
This figure "fig2-2.png" is available in "png" format from: http://arxiv.org/ps/hep-ph/9510223v1 
This figure "fig1-3.png" is available in "png" format from: http://arxiv.org/ps/hep-ph/9510223v1 
This figure "fig2-3.png" is available in "png" format from: http://arxiv.org/ps/hep-ph/9510223v1 